\title{
Research Note \\ Th4-4: A Be star which has turned into a planetary nebula within 30 years
}

\begin{abstract}
L. N. Kondratyeva
Fesenkov Astrophysical Institute, Kazakhstan, Isaak Newton Institute of Chile, Kazakhstan Branch, 480020 Almaty Observatory, Kazakhstan

Received 8 January 2001 / Accepted 20 June 2001

Abstract. The first optical spectra of Th4-4, obtained by the author in 1970, showed strong continuum and broad HI emission lines. In other words, at this time the object looked like a Be star. Some years later, [OIII] emission lines appeared in the spectrum indicating the existence of a gaseous envelope. Today the optical spectrum of Th4-4 is quite similar to that of a moderate excitation planetary nebula. The magnitude of the object has dropped from $V=14^{\mathrm{m}} \cdot 4$ to $16{ }^{\mathrm{m}} 6$, and the effective temperature of the central star has increased from $22000 \mathrm{~K}$ up to $40000 \mathrm{~K}$ within the last 30 years.
\end{abstract}

Key words. planetary nebulae: general: early type stars - stars: emission lines, Be stars: individual: Th4-4- stars: evolution

\section{Introduction}

Some new information appeared recently about objects showing rapid change from an early type star to something looking like a planetary nebula (Parthasarathy et al. 1993; Smith \& Lambert 1994). The most intriguing fact is that such a transformation takes only some decades too short a time to really allow evolution modification. Nevertheless, as a result, we have objects which look like planetary nebulae, but their real nature is unknown.

Th4 $-4\left(\mathrm{PK} 8+3^{\circ} 2=\mathrm{PNG} 8.3+03.7=\mathrm{ARO} 260=\right.$ ESO589 - 10) with the coordinates $(2000): 17^{\mathrm{h}} 50^{\mathrm{m}} \cdot 4$ $19^{\circ} 54$, is one of these objects. Discovered by The (1964), it was listed in the Catalogue of Galactic Planetary Nebulae of Perek \& Kohoutek (1967). Then, in 1970, it was classified as a Be star due to a strong continuum and broad HI emission lines (Kondratyeva 1975). Further spectral observations of Th4-4 have shown [OIII] emission lines, and in 1981 the object was classified as a low-excitation planetary nebula (Kondratyeva 1989). Allen (1984) considered this object to be symbiotic with a $\mathrm{M}$ star continuum and bright emission lines of $\mathrm{HI}$, HeI, and [ArIII]. A ten-minutes exposure spectrum, obtained in July 1986, shows only a faint continuum; therefore the object was classified as "possible PN" (Acker et al. 1992). Observations of Gutierrez-Morreno (1992) have confirmed that Th4-4 is a low-excitation planetary

Send offprint requests to: L. N. Kondratyeva, e-mail: Kon@afi.south-capital.kz nebula. Multicolor measuring was carried out by Munari et al. (1992). Regular optical observations of this object were conducted since 1970 at the Fesenkov Astrophysical Institute (Almaty, Kazakhstan). During 1970-1990 the light of Th4-4 has weakened by a factor of 7.5, and the intensities of some emission lines ([OIII], He1, HeII) have increased (Kondratyeva 1989). This paper presents new observational results, obtained during the last ten years.

\section{Observations and results}

Spectral and photometric observations of Th4 4 have been carried out by the author with the $70-\mathrm{cm}$ telescope of Astrophysical Institute (Almaty, Kazakhstan).

\subsection{Spectral observations}

We used a slit spectrograph, equipped with a threecascade image tube until 1998, and with a CCD matrix ST7 since 1999. A sample of gratings and objective lenses provided a spectral range from 3700 to $8200 \AA$, a dispersion in the range of $20-200 \AA / \mathrm{mm}$, with a spectral resolution $\Delta \lambda$ equal to $1.2-10 \AA$. Wavelength calibration has been done using a laboratory source of HeI, Ne and Ar emission lines. Flux calibration was performed through observations of standard stars ( $\zeta \mathrm{Tau}, 62 \mathrm{Tau}, 55 \mathrm{Cyg}, \zeta \mathrm{Cas}$ ) (Kharitonov et al. 1978).

During the last ten years, 10 spectra of Th4-4 were obtained, as listed in Table 1a. All spectra were measured, 
Table 1a. Journal of spectral observations of Th4-4.

\begin{tabular}{|c|c|c|c|c|}
\hline Date & $\begin{array}{l}\text { Spectral range } \\
(\AA)\end{array}$ & $\begin{array}{c}\text { Spectral } \\
\text { Resolution } \\
\AA\end{array}$ & $\begin{array}{c}\text { Exposure } \\
\text { times } \\
(\min )\end{array}$ & $\begin{array}{l}\text { Number of } \\
\text { spectra }\end{array}$ \\
\hline July 1991 & $4300-5100$ & 2.5 & 45 & 2 \\
\hline Aug. 1991 & $5850-7300$ & 5.0 & 30 & 1 \\
\hline June 1994 & $4300-5100$ & 2.5 & 55 & 1 \\
\hline May 1995 & $4300-5100$ & 2.5 & 60 & 2 \\
\hline June 1998 & $4300-5100$ & 2.5 & 50 & 1 \\
\hline June 1998 & $5950-7300$ & 5.0 & 30 & 1 \\
\hline June 1999 & $4300-5100$ & 2.5 & 55 & 1 \\
\hline May 2000 & $4300-5100$ & 2.5 & 60 & 1 \\
\hline
\end{tabular}

Table 1b. Journal of photometric observations of Th4-4.

\begin{tabular}{lcc}
\hline Date & $\begin{array}{c}\text { Number } \\
\text { of images }\end{array}$ & $V$ mag \\
\hline July 1991 & 6 & $16,43 \pm 0.09$ \\
Aug. 1991 & 6 & $16,40 \pm 0.08$ \\
June 1994 & 8 & $16,25 \pm 0.07$ \\
May 1995 & 5 & $16,22 \pm 0.09$ \\
June 1998 & 7 & $16,24 \pm 0.07$ \\
June 1999 & 5 & $16,15 \pm 0.09$ \\
May 2001 & 3 & $16,03 \pm 0.06$ \\
\hline
\end{tabular}

but the intensity is reliable only for the strongest emission lines, as the object was very faint. The precision of data obtained is $10 \%, 15 \%$ and $50 \%$ for moderate, strong and faint lines respectively.

Spectral variations were observed. The intensities of [OIII] lines (relative to $\mathrm{H} \beta$ ) increased by $20 \%$ during $1992-1994$ and only by $1-2 \%$ over the last 6 years. HeII emission lines strengthened around 2\% within 10 years. Relative intensities of emission lines, observed in 1998-2000, are given in Table 2. All values were dereddened using $c(\mathrm{H} \beta)=2.13$ (Acker et al. 1991). Figures 2c,d present two fragments of averaged spectra, obtained in 1998-2000. Neither traces of continuum nor [NII] or [SII] emission were detected.

\subsection{Photometric observations}

Photometric observations of Th4-4 were carried out simultaneously with the spectral observations $(2-5$ images by night). Direct images were obtained with the threecascade image tube, attached to the $70-\mathrm{cm}$ telescope, and using a color system with a passband $\approx 800 \AA$, centered at $\lambda 5460 \AA$, close to the standard $V$-band. The size of the projected field of sky was equal to $150^{\prime} \times 150^{\prime}$, the scale of image was about $40^{\prime \prime}$ per mm. Exposure times of 10-15 s. were sufficient to obtain good quality images of stars up to 17 mag. Seven stars near to the studied object were chosen to be secondary standards; their visual magnitudes covered rather a large range: $12-17$ mag. The intrinsic errors
Table 2. Line intensity ratios for Th4-4 (1998-2000).

\begin{tabular}{lc|lc}
\hline Line & $\begin{array}{c}\text { Flux } \\
(\mathrm{H} \beta=100)\end{array}$ & Line & $\begin{array}{c}\text { Flux } \\
(\mathrm{H} \beta=100)\end{array}$ \\
\hline $4686 \mathrm{HeII}$ & $10.8 \pm 1.8$ & $5876 \mathrm{HeI}$ & $15 \pm 2.0$ \\
$4861 \mathrm{H} \beta$ & $100 \pm 3.1$ & $6563 \mathrm{H} \alpha$ & $296 \pm 22$ \\
$4921 \mathrm{HeI}$ & $7.4 \pm 1.5$ & $6678 \mathrm{HeI}$ & $9 \pm 3.0$ \\
$4959[\mathrm{OIII}]$ & $61.7 \pm 4.5$ & $7065 \mathrm{HeI}$ & $12 \pm 2.1$ \\
$5007[\mathrm{OIII}]$ & $191 \pm 10$ & & \\
\hline
\end{tabular}

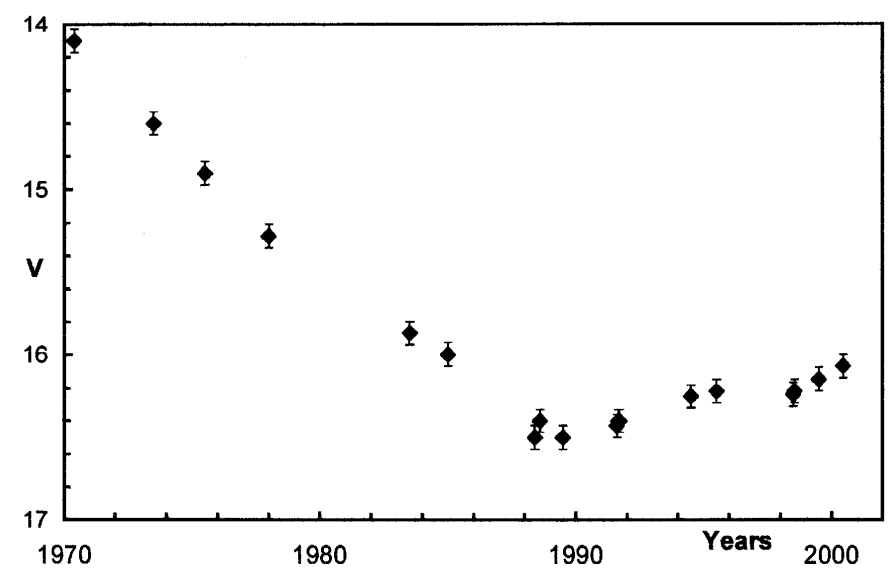

Fig. 1. Light curve of Th4-4.

of photometry were equal to $0{ }^{\mathrm{m}} 03-0{ }^{\mathrm{m}} 07$ depending on the star's magnitudes. Differential photometry of Th4-4 relatively to the comparison stars in the same image was obtained. All these primary estimations of magnitude were related to "our" color system (Mobs). We estimated that $\Delta$ Mobs $=\Delta \mathrm{V}$. Then using $V=16.40 \pm 0.04$, obtained by Munari et al. (1992), and our Mobs value for the closed date of observations, we transformed our sample of data into the $V$ color system. Final results with their errors are presented in the last column of Table $1 \mathrm{~b}$.

The light curve of Th4-4 covering 30 years is presented in Fig. 1. One can see that the brightness rapidly decreases by 2.5 magnitudes from 1970 to 1990 . Then the star becomes slightly brighter, but during the period 1991-2000 the variation did not exceed $0.2 \mathrm{mag}$.

\section{Discussion}

Let us review the photometric and spectral history of Th4-4. In 1970-1971 the object looked like a star of $V=14^{\mathrm{m}} 10 \pm 0.05$ (Kondratyeva 1989). Some information about its earlier magnitude can be obtained using images of the object taken in 1960 in the framework of the Palomar Survey. We derived a rough value $V=14^{\mathrm{m}} 3 \pm 0 .{ }^{\mathrm{m}} 6$. Thus it may be supposed that "bright" phase of Th4-4 lasted at least 10 years and stopped in 1972-1973. During this stage the object shows a spectrum with a strong continuum, broad $\mathrm{H} \alpha$ and $\mathrm{H} \beta$ emission lines, weak HeI emission lines and numerous weak FeI 
absorption lines (Fig. 2a). The profile of $\mathrm{H} \alpha$ shows a width $(F W H M)$ equal to $5.5 \pm 0.2 \AA$, and wings extending over $\approx 10 \AA$, implying a radial speed up to $450 \mathrm{~km} \mathrm{~s}^{-1}$. The object was classified as a Be star (Kondratyeva 1975). The value of the effective temperature $T_{\text {eff }}=22000 \mathrm{~K}$ was determined.

During the next 15 years (1975-1989) rapid weakening of light and spectral changes were observed. Since 1975, forbidden lines of [OIII] 4363, 4959, $5007 \AA$ appeared with increasing intensities (Kondratyeva 1989; Acker et al. 1991; Gutierrez-Morreno et al. 1992a) (see Fig. 2b).

For their spectrum taken in 1987, Acker et al. (1991) derived a very low excitation class; the energy-balance method leads to a stellar temperature of $35000 \mathrm{~K}$ (PreiteMartinez et al. 1989).

In 1990 Th4-4 entered in a rather stable phase. It seems that the decrease in visual luminosity of the object had almost stopped or even began to increase in brightness. Further amplification of HeII and [OIII] emission is under observations, but it has become quite slow (see Figs. 2b,c).

What is the true nature of this object? Photometric and spectral behavior of Th4-4 may be the result of a quickly expanding dense envelope, ionized by stellar radiation. Two observational facts show the existence of a late type component: an M-type continuum was observed by Allen (1984), and dereddened infrared color indices (Munari 1990) place Th4-4 on the red giant branch on the $(J-H)$ vs. $(H-K)$ diagram. Moreover, the light curve of Th4-4 is similar to those of some symbiotic slow novae, such as RRTel and RTSer (Murset 1994). In particular, the amplitude and weakening rate of Th4-4 are quite the same as those of RRTel. Thus we can consider Th4-4 as a binary symbiotic system after an outburst. However, some differences in spectra and in stellar temperatures are observed. It is known that the temperature of ionizing radiation of symbiotic nova after outburst increases up to very high values due to the compression of accreted star (Iben \& Tutukov 1996). For example, when RRTel entered in a steady phase, its temperature was equal to $140000 \mathrm{~K}$, and the highest ionization energy of the observed spectrum exceeded $100 \mathrm{eV}$ (Murset 1994). The highest ionization potential observed recently for Th4-4 is $54 \mathrm{eV}$. Effective temperature of ionizing radiation is estimated to be $40000-45000 \mathrm{~K}$ by using the [OIII] $5007 / \mathrm{H} \beta$ lines ratio (Kaler 1991).

\section{Conclusion}

Observational data, obtained over 30 years, allow us to follow the history of Th4-4, and to compare the object with a symbiotic nova. Recent spectroscopic observations lead us to classify the object as a planetary nebula without any doubt, but some transformations can be expected in the future. Further investigations are necessary in order to study all the possible spectral and photometric variations of Th4-4. This time we don't know anything about its structure and angular size. Observations with more
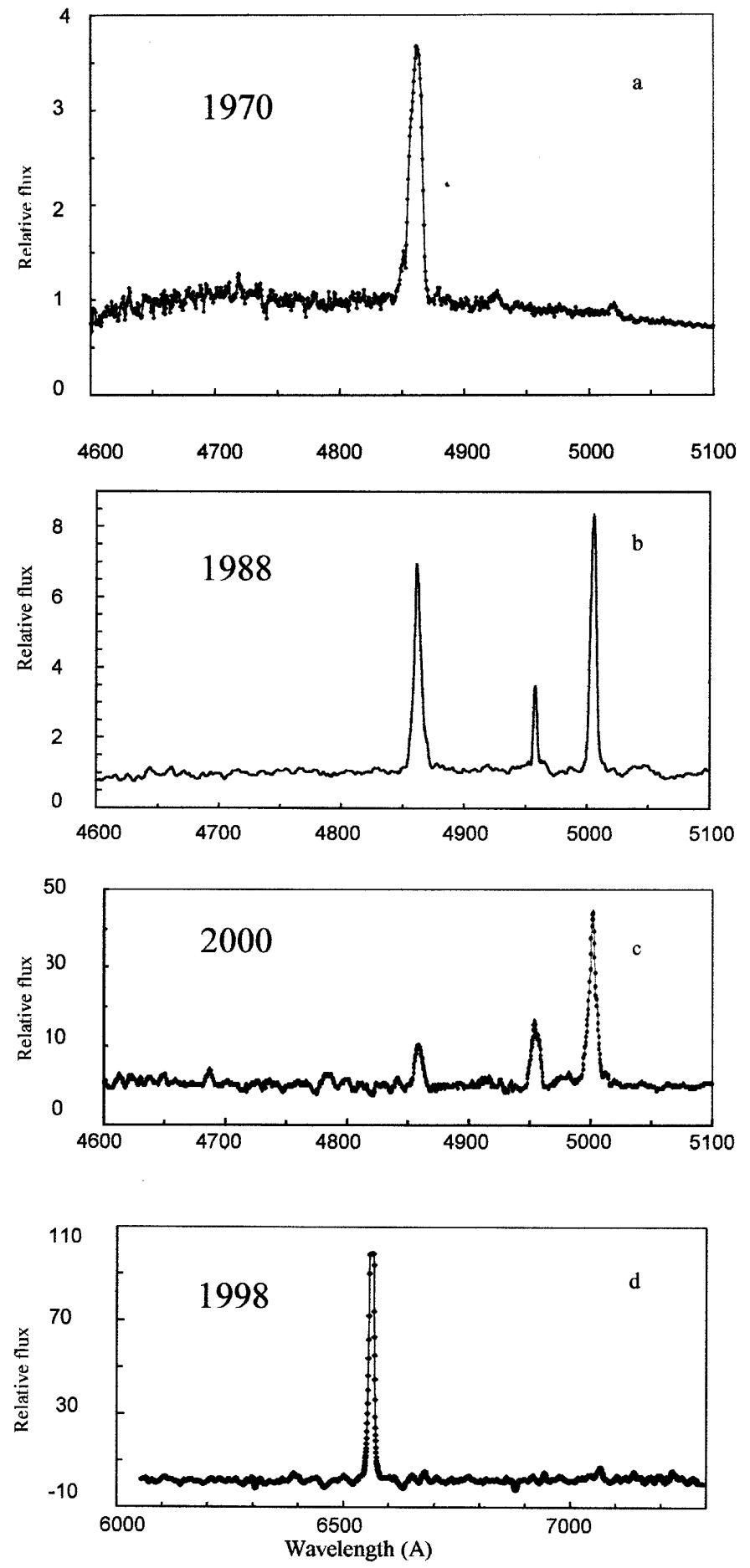

Fig. 2. Spectrum of Th4-4. Panels a,b): Observational data obtained in 1970 (May-June) and in 1988 (June-July). Intensities are normalized to the intensity of continuum at $\lambda 4861$ A. Panels c,d): Spectral data, obtained in 1998-2000, are represented in arbitrary relative units.

powerful instruments should be needed in order to obtain full information about the visual and near infrared spectrum of Th4-4. 


\section{References}

Acker, A., et al. 1991, A\&AS, 89, 237

Acker, A., Ochsenbein, F., et al., 1992, The Strasbourg-ESO Catalogue of galactic planetary nebulae

Allen, D. 1984, Proc. Astron. Soc. Australia, 5, 367

Garsia-Larto, P., Machado, A., et al. 1993, A\&A, 267, L11

Gutierrez-Morreno, A., \& Cortes, G. 1992a, PASP, 104, 1187

Gutierrez-Morreno, A., et al. 1992b, A\&AS, 93, 38

Iben, I. Jr., \& Tutukov, A. V. 1996, A\&AS, 105, 145

Kaler, J. B. 1991, ApJ, 372, 215

Kharitonov, A. V., Tereschenko, V. M., \& Knjazeva, L. N. 1978, Spectrophotometric Catalogue of Stars, Alma-Ata, Kazakhstan
Kondratyeva, L. N. 1975, Trudy of AFI (Kazakhstan), 25, 23 Kondratyeva, L. N. 1989, Sov. Astron Lett., 15, 29 Munari, U., Yudin, B. F., et al. 1992, A\&AS, 93, 388 Murset, U., \& Nussaumer, H. 1994, A\&A, 282, 586 Parthasarathy, M., Garcia-Larto, P., et al. 1993, A\&A, 267, L19

Parthasarathy, M., \& Pottash, S. R. 1986, A\&A, 154, L16

Parthasarathy, M. 1990, Proc. IAU Symp., 145, 119

Perek, L., \& Kohoutek, L. 1967, Catalogue of galactic Planetary nebulae, Prague

Preite-Martinez, A., Acker, et al. 1989, A\&AS, 81, 309

Smith, V., \& Lambert, D. 1994, ApJ, 424, L123

The P. S. 1964, Contr. Bosscha Obs., 28, 5 